\title{
Prevalence of high-risk coronary plaques in patients with and without metabolic syndrome and the relationship with prognosis
}

\author{
Xu Yang ${ }^{1}$, Wei Luo ${ }^{1}$, Shan Han ${ }^{1}$, Lei Zha', Jing Zhang ${ }^{1}$, Xiaowei Li ${ }^{1}$, Hui Zhao ${ }^{2}$, Shuo Liang ${ }^{2}$ and Ru Zhao ${ }^{1 *}$ (D)
}

\begin{abstract}
Background: Metabolic syndrome (MS) is a disorder, characterized by clusters of cardiovascular risk factors such as central obesity, insulin resistance, dyslipidemia and hypertension. Patients with MS may have a higher plaque burden that increases their risk of major adverse cardiovascular events (MACEs). This study aimed to analyze the prevalence of high-risk coronary plaques in patients with and without MS by coronary computed tomography angiography (CCTA) and to investigate the relationship between MS, high-risk coronary plaques, and their prognosis.

Methods: This was a retrospective cohort study of 1136 patients who underwent CCTA due to chest pain without obstructive heart disease ( $\geq 50 \%$ coronary stenosis) between January 2014 and December 2015 in our hospital. The relationships between high risk coronary plaques, MS, and other clinical factors were assessed. Multicollinearity analysis was performed to identify the collinearity between the variables. The proportional hazard assumption was checked and using Schoenfeld residual test. Cox proportional hazards model and Kaplan-Meier survival analysis assessed the relationship between MS, high-risk coronary plaques and MACEs.

Results: High-risk plaques were more frequent in the MS group than non-MS group $(P=0.004)$. MS $(H R=2.128,95 \% \mathrm{Cl}$ : $1.524-2.970, P<0.001)$, presence of high-risk plaques ( $\mathrm{HR}=11.059,95 \% \mathrm{Cl}: 7.749-57.232, P<0.001)$ and high sensitivity C-reactive protein (hsCRP) $(H R=1.629,95 \% \mathrm{Cl}: 1.128-2.352, P=0.009)$ were related with an increased risk of MACEs in patients with risk factors for coronary heart disease. In patients with high-risk plaques, MS (HR=2.265, 95\%Cl: 1.629$3.150, P<0.001)$ and hsCRP ( $H R=1.267,95 \% \mathrm{Cl}: 1.191-1.348, P=0.004)$ were related with an increased risk of MACEs. Kaplan-Meier analysis showed differences in MACEs between the MS and non-MS groups in the whole population and those with high-risk plaques (both $P<0.0001$ ).

Conclusions: High-risk plaques were more common in patients with MS. MS and the presence of high-risk plaques were independent risk factors for MACEs.
\end{abstract}

Keywords: Metabolic syndrome, Atherosclerosis, Risk factors, Prognosis

\footnotetext{
* Correspondence: heart6207@sina.com

'Department of Cardiology, Tianjin Chest Hospital, Tianjin 300222, People's

Republic of China

Full list of author information is available at the end of the article
}

(c) The Author(s). 2020 Open Access This article is distributed under the terms of the Creative Commons Attribution 4.0 International License (http://creativecommons.org/licenses/by/4.0/), which permits unrestricted use, distribution, and reproduction in any medium, provided you give appropriate credit to the original author(s) and the source, provide a link to the Creative Commons license, and indicate if changes were made. The Creative Commons Public Domain Dedication waiver (http://creativecommons.org/publicdomain/zero/1.0/) applies to the data made available in this article, unless otherwise stated. 


\section{Background}

Metabolic syndrome (MS) occurs when an individual has a few risk factors, such as central obesity, insulin resistance, dyslipidemia and hypertension that together result in an increased risk of type 2 diabetes mellitus and cardiovascular disease [1]. In China, the prevalence of MS is around $33.9 \%$ overall with a prevalence of $31.0 \%$ in men and $36.8 \%$ in women [2]. As well as an increased risk of cardiovascular disease MS is also implicated in increased risk of sudden cardiac death [3]. Among the reasons for this is that factors associated with MS may increase coronary calcification and subclinical atherosclerosis (AS) [4].

AS may predict major adverse cardiac events (MACEs), a high-risk coronary plaque may progress rapidly and result in acute coronary syndrome [5]. Coronary computed tomography angiography (CCTA) can not only effectively evaluate the degree of coronary artery stenosis, but also analyze the coronary atherosclerotic plaque characteristics to identify high-risk plaques, which has unique advantages compared to other examinations [6]. CCTA studies used to mainly focus on distinguishing vessels by their degree of coronary stenosis. But in a histopathological study of ruptured plaques, $30 \%$ produced of nonobstructive ruptured plaques were further subdivided into those with luminal narrowing of $50-75 \%$ and those with luminal narrowing < $50 \%$ ( 25 and $5 \%$ of lesions respectively) [7]. Before the occurrence of acute coronary events, such patients often miss their optimal diagnosis time and treatment due to the lack of symptoms or the absence of severe stenosis of the coronary lumen. Therefore, detection of high-risk plaques may effectively reduce the occurrence of MACEs [8].

Identification of high-risk plaque features has been in the focus of coronary CTA imaging in the past years [9]. At present, positive remodeling, low computed tomography $(\mathrm{CT})$ attenuation, napkin ring sign, and spotty calcification are all considered to be characteristics of highrisk plaques $[6,10]$, but the risk factors for rapid progression of high-risk plaques to MACEs are still unclear. MS is a complex disorder [11], and the factors involved aggregate inflammatory reactions in individuals and increases plaque instability [12]. A few studies have shown that patients with MS have a higher plaque burden and increase the risk of future occurrence of acute cardiovascular events and poor prognosis [10, 13-16].

The aim of the present study was to investigate the prevalence of high-risk coronary plaques in patients with and without MS and then to study the relationship between MS, high-risk plaques and prognosis.

\section{Methods}

\section{Patients}

This was a retrospective cohort study. Patients who underwent CCTA due to chest pain in our hospital from January 2014 to December 2015 were retrospectively analyzed. The inclusion criteria were: 1) Age $\geq 18$ years old; 2) Patients who had chest pain and underwent CCTA; 3) Patients who had two or more risk factors of coronary heart disease (male > 45 years old, female $>55$ years old; history of smoking; history of diabetes; high-density lipoprotein $<1.0 \mathrm{mmol} / \mathrm{L}$; history of hypertension; family history of coronary heart disease occurred in $<55$ years old males or $<65$ years old women in first-degree relatives).

The exclusion criteria were as follows: 1) Patients who had a history of coronary revascularization or more than $50 \%$ stenosis of crosssectional luminal area shown by CCTA. 2) Patients who had arrhythmia, severe cardiac dysfunction (NYHA $\geq$ III degree); 3) Renal insufficiency (serum creatinine clearance rate, Scr $>120 \mathrm{ml} / \mathrm{min}$ ); 4) Hyperthyroidism; 5) Contrast agent allergy history or the iodine allergy test was positive; 6) Poor quality of CCTA images.

This study was approved by the Institutional Review Board of our institution (the ethics number:2019LW006). All patients underwent multidetector CT and had provided written informed consent after they agreed to participate in our study.

\section{Grouping}

In accordance with the modified National Cholesterol Education Program-Adult Treatment Panel III criteria, an individual was defined as having MS if he or she had three or more of the following five criteria $[17,18]$ : (1) Waist circumference $\geq 90 \mathrm{~cm}$ in men and $\geq 80 \mathrm{~cm}$ in women using the International Obesity Task Force criteria for the Asian-Pacific population to determine waist circumference criteria; (2) Triglyceride levels $\geq 1.7 \mathrm{mmol} /$ $\mathrm{L}$; (3) HDL-cholesterol level $<0.9 \mathrm{mmol} / \mathrm{L}$ in men and $<$ $1.0 \mathrm{mmol} / \mathrm{L}$ in women; (4) Blood pressure $\geq 130 / 85$ $\mathrm{mmHg}$ or the use of antihypertensive medication; and (5) Fasting glucose level $\geq 6.1 \mathrm{mmol} / \mathrm{L}$ or the selfreported use of antidiabetic medication (insulin or oral agents). According to the above diagnostic criteria, the number of risk factors for metabolic syndrome in each patient was counted.

\section{CCTA examination}

CCTA were conducted with dual source CT (SOMATOM DEFINITION, Siemens). Overlapping 64-layer images with a layer thickness of $0.6 \mathrm{~mm}$ were obtained. Before the CCTA examination, the process was explained to the patient so that they could cooperate with the examination during the scan. The scanning room was equipped with various rescue drugs, rescue equipment and oxygen. All patients underwent an iodine allergy test prior to the test. Patients who had heart rate > 70 beats/min were given an oral beta-blocker $30 \mathrm{~min}$ before CT coronary angiography.

During the examination, the patient's anterior elbow vein was injected with a double-tube high-pressure 
syringe at a flow rate of $3.5-4.5 \mathrm{ml} / \mathrm{s}$ according to the patient's weight. The contrast agent was Ultravist $(370 \mathrm{mg}$ I/100 ml, Bayer Health Care Co., Ltd) or Iohexol (350 mg I/100 ml, General Electric Pharmaceuticals). The scan range was $10-15 \mathrm{~mm}$ below the tracheal divergence to the palpebral surface $(120 \mathrm{kV}, 100 \mathrm{mAs})$ and the scan time was $6-10 \mathrm{~s}$. Then, a coronary enhancement scan was performed, and the scanned images were processed and screened by multi-layer reconstruction (MPR), curved planar reconstruction (CPR), maximum intensity projection (MIP), volume rendering (VR) and cardiovascular optimization analysis software. Optimal CT images were used for the evaluation of coronary vascular plaques.

\section{CCTA image analysis}

The coronary arteries were divided into 16 segments according to the American Heart Association classification method [19], and images were analyzed independently by 2 radiologists engaged in cardiovascular imaging diagnosis. When the results of the two doctors' evaluations were inconsistent, they were read again and the opinions were unified. If their opinions were still not unified, another senior radiologist was engaged.

Coronary plaques were defined as having an area greater than 1 square millimeter visible at least in two mutually perpendicular orientations [20]. Obstructive coronary stenosis was defined as a coronary plaque causing luminal stenosis $\geq 50 \%$. If CCTA did not show any coronary plaque (including calcification) the scan was defined as normal, if there was a plaque in any coronary artery with stenosis of $<50 \%$ this was defined as mild stenosis, if there was a plaque in any coronary artery resulting in $50-69 \%$ stenosis this was defined as moderately stenotic, a plaque resulting in stenosis of $\geq 70 \%$ was defined as severe stenosis [21, 22].

As shown in Fig. 1, a high-risk plaque had at least one of the following characteristics [6, 20]: (1) Spotty calcification: defined as the presence of calcification with a diameter $<3 \mathrm{~mm}$ in the CCTA field, the length did not exceed 1.5 times the diameter of the lumen, and the width did not exceed $2 / 3$ of the lumen diameter. (2) Low attenuation: plaque attenuation was assessed using a Hounsfield Unit (HU). If the average CT value of 3 interest areas randomly selected around the noncalcified plaque (the range is about $0.5-1.0 \mathrm{~mm}^{2}$ ) was < $30 \mathrm{HU}$, the plaque was defined as low attenuation. (3) Positive remodeling: defined as the arterial remodeling index ( $\mathrm{RI}=$ lesion plaque area/reference area) being greater than or equal to 1.1 (Reference segment: the same blood vessel, narrow and proximal $10 \mathrm{~mm}$, where the lumen area was the largest, and the parts without major branches were defined as the proximal reference segment and the distal reference segment respectively). (4) Napkin ring sign: the central low attenuation lesion connected with the cavity was surrounded by a ring with slightly higher attenuation plaque tissue.

\section{Clinical data collection and follow-up}

The general information of all subjects such as age, gender, body mass index (BMI), history of diabetes, hypertension, hyperlipidemia, blood pressure, fasting blood glucose (FBG), triglycerides (TG), low-density lipoprotein cholesterol (LDL), high-density lipoprotein cholesterol (HDL) and high sensitivity C-reactive protein (hsCRP) were collected. Furthermore, pharmacological treatment of hypertension, diabetes, hyperlipidemia and application of aspirin and statins were also
A
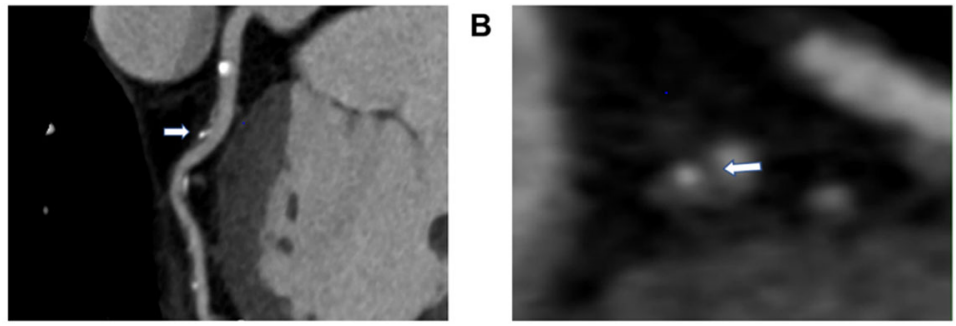

C

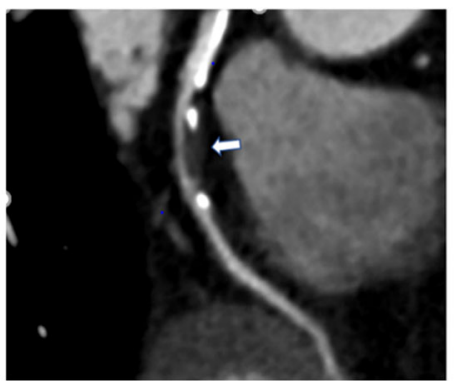

D

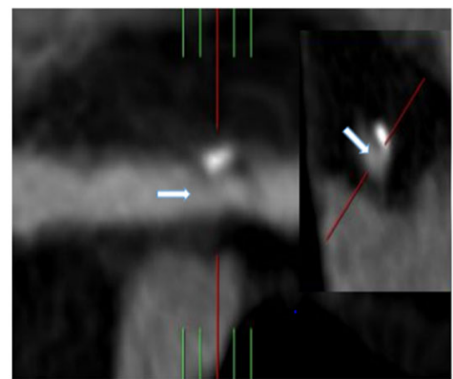

Fig. 1 Characteristics of high-risk plaques (white arrow). a Spotty calcification; b Low attenuation; c Positive remodeling; d Napkin ring sign 
recorded. During follow-up, a lifestyle of light diet, smoking cessation, alcohol restriction and moderate exercise is recommended.

Follow-up observation: the patients were followed up once every month for a period of 36 months. The pharmacological treatment of hypertension, diabetes, hyperlipidemia and application of aspirin and statins during the follow-up period were recorded unceasingly. The end point was the occurrence of MACEs including cardiac death, nonfatal myocardial infarction, and hospitalization for unstable angina or revascularization. Cardiac death was defined as death caused by acute myocardial infarction, ventricular arrhythmia, or refractory heart failure. Nonfatal myocardial infarction was defined as both ST-elevation myocardial infarction (STEMI) and unstable angina/non-STEMI (UA/NSTE-MI). Unstable angina was recorded as an event only in case urgent hospital admission was required.

\section{Statistical analysis}

SPSS17.0 (SPSS Inc., Chicago, IL, USA) was used for all statistical analyses except proportional hazard assumption was checked by STATA 15.0 (StataCorp LLC StataCorp). Student's t tests or Mann-Whitney U test were used for comparing continuous variables and $\chi^{2}$ tests for categorical variables. All data were expressed as the mean \pm standard deviation (SD), median (interquartile range), or number (frequency). Possible multicollinearity was quantified using the variance infiltration factor (VIF). A VIF > 4.0 was considered an indication of harmful multicollinearity in the regression model. A Cox proportional hazard model was used to investigate any independent effect of MS, each individual MS component on the MACEs by adjusting significant variables. The proportional hazard assumption was checked using Schoenfeld residual tests. Kaplan-Meier survival analysis assessed the relationship between MS, high-risk coronary plaques and MACEs. All the tests were bilateral and $P<0.05$ was considered statistically significant.

\section{Results}

\section{Baseline characteristics}

A total of 1136 patients were included, 488 were in the MS group and 648 in the non-MS group. Typical characteristics of high-risk plaques are shown in Fig. 1. There was no significant difference in age $(P=0.787)$ and gender $(P=0.857)$ between the two groups. Pharmacological treatment of hypertension $(P=0.518)$, diabetes $(P=0.079)$, hyperlipidemia $(P=0.201)$, aspirin $\quad(P=$ $0.147)$ and statins $(P=0.093)$ also showed no significant difference between the two groups. The BMI of the MS group was significantly higher than that of the non-MS group $\left(26.6 \pm 3.4 \mathrm{~kg} / \mathrm{cm}^{2}, \quad 25.2 \pm 3.3 \mathrm{~kg} / \mathrm{cm}^{2}, \quad P=0.001\right)$. Waist circumference was higher in the MS group compared to the non-MS group $(93.7 \pm 6.8 \mathrm{~cm}$ vs. $88.3 \pm 6.3$ $\mathrm{cm}, P<0.001)$. There were higher rates of MACEs and high-risk plaques in the MS compared to the non-MS group $(27.5 \%$ vs. $11.7 \%, P<0.001 ; 66.8 \%$ vs. $59.3 \%, P=$ 0.004).(Table 1).

\section{Characteristics of high-risk plaque analysis}

Table 2 presents the data when only the 704 patients with high-risk plaques were considered (326 in the MS group and 378 in the non-MS group). There was no significant difference in age $(P=0.288)$ and gender $(P=0.577)$ between the two groups. Pharmacological treatment of hypertension $(P=0.501)$, diabetes $(P=0.069)$, hyperlipidemia $(P=0.132)$, aspirin $(P=0.112)$, and statins $(P=0.231)$ also showed no significant difference between the two groups. The hsCRP levels in the MS group were significantly higher than those in the non-MS group, and the differences were statistically significant $(8.0 \pm 2.7 \mathrm{mg} / \mathrm{l}$ vs. $7.1 \pm 3.2 \mathrm{mg} / \mathrm{l}, \mathrm{P}<0.001)$. In terms of the imaging characteristics of high-risk plaques in the two groups, the ratio of positive remodeling, spot-like calcification and napkin ring signs in the MS group were significantly higher than that in the non-MS group, and the difference was statistically significant $(66.3 \%$ vs. $54.2 \%, P=0.001 ; 65.3 \%$ vs $54.8 \%, P=0.004 ; 50.6 \%$ vs. $40.7 \%, P=0.010)$.

\section{Follow-up}

All the patients were followed up by telephone and outpatient service for 36 months. All Patients were encouraged to practice heart-healthy lifestyle behaviors [23], including: (1) Consume a dietary pattern that emphasizes intake of vegetables, fruits, and whole grains; includes low-fat dairy products, poultry, fish, legumes, nontropical vegetable oils, and nuts; (2) Limits intake of sodium, sweets, sugar-sweetened beverages, and red meats. (3) Counseling to reduce sodium intake by an average of $1150 \mathrm{mg} / \mathrm{d}$. Engage in $2 \mathrm{~h}$ and $30 \mathrm{~min}$ per week of moderate-intensity physical activity. With a loss rate of 6.1\%, 30 patients in the MS group and 48 patients in the non-MS group were lost to follow-up. Finally, 144 patients in the MS group had MACEs during the follow-up period $(27.5 \%)$, while 76 patients in the non-MS group had MACEs during the follow-up period (11.7\%), and the difference was statistically significant $(P<0.001)$. For those who had high risk plaques, 96 patients in the MS group had MACEs during the follow-up period (29\%), while 57 patients in the non-MS group had MACEs during the follow-up period (15\%), and the difference was statistically significant $(P<0.001)$. (Tables 1 and 2$)$.

COX regression analysis was used to adjust for variables had statistical difference in Table 1, such as age, sex, BMI, waist circumference, LDL, TG, and FBG. The proportional hazard assumption was checked by the Schoenfeld residual global test, and there was no breach of that hypothesis $(P=0.159)$. Variance inflation factors 
Table 1 Baseline clinical and biochemical characteristics in the MS group and non-MS group

\begin{tabular}{|c|c|c|c|c|}
\hline & MS $(n=488)$ & Non-MS $(n=648)$ & $P$ & VIF \\
\hline Age (years), mean $\pm S D$ & $61.5 \pm 9.29$ & $61.6 \pm 8.70$ & 0.787 & 1.056 \\
\hline Male, n (\%) & $240(49.2)$ & $323(49.8)$ & 0.857 & 1.125 \\
\hline BMI $\left(\mathrm{kg} / \mathrm{cm}^{2}\right)$, mean \pm SD & $26.6 \pm 3.43$ & $25.2 \pm 3.31$ & 0.001 & 2.820 \\
\hline Waist circumference $(\mathrm{cm})$, mean \pm SD & $93.7 \pm 6.80$ & $88.3 \pm 6.34$ & $<0.001$ & 2.780 \\
\hline Hypertension, n (\%) & $307(62.9)$ & $369(56.9)$ & 0.043 & 1.348 \\
\hline Diabetes, n (\%) & $306(57.8)$ & $425(34.4)$ & $<0.001$ & 1.275 \\
\hline Hyperlipidemia, n (\%) & $276(56.6)$ & $261(40.3)$ & $<0.001$ & 2.875 \\
\hline $\mathrm{FBG}(\mathrm{mmol} / \mathrm{L})$, mean $\pm \mathrm{SD}$ & $6.5 \pm 3.48$ & $6.4 \pm 3.66$ & 0.046 & 1.071 \\
\hline LDL (mmol/l), mean \pm SD & $3.5 \pm 0.76$ & $3.2 \pm 0.87$ & $<0.001$ & 2.725 \\
\hline $\mathrm{TG}(\mathrm{mmol} / \mathrm{l})$, mean $\pm \mathrm{SD}$ & $1.8 \pm 1.43$ & $1.5 \pm 0.92$ & 0.001 & 1.070 \\
\hline $\mathrm{HDL}(\mathrm{mmol} / \mathrm{l})$, mean $\pm \mathrm{SD}$ & $0.9 \pm 0.69$ & $1.0 \pm 0.69$ & 0.165 & 1.940 \\
\hline hsCRP (mg/l), mean $\pm \mathrm{SD}$ & $8.0 \pm 2.72$ & $7.1 \pm 3.15$ & $<0.001$ & 1.417 \\
\hline \multicolumn{5}{|l|}{ Pharmacological treatment, n (\%) } \\
\hline Hypertension, n (\%) & $209(42.9)$ & $190(44.8)$ & 0.518 & 1.126 \\
\hline Diabetes, n (\%) & $187(38.5)$ & $217(33.5)$ & 0.079 & 1.193 \\
\hline Hyperlipidemia, n (\%) & $127(26.1)$ & $191(29.5)$ & 0.201 & 1.423 \\
\hline Aspirin, n (\%) & $223(45.7)$ & $268(41.4)$ & 0.147 & 1.106 \\
\hline Statins, n (\%) & $116(23.8)$ & $127(19.6)$ & 0.093 & 1.415 \\
\hline Presence of high-risk plaque, n (\%) & $326(66.8)$ & $378(58.3)$ & 0.004 & 2.998 \\
\hline MACEs, n (\%) & $144(27.5)$ & $76(11.7)$ & $<0.001$ & \\
\hline
\end{tabular}

MS metabolic syndrome; $B M I$ body mass index; $F B G$ fasting blood glucose; $L D L$ low-density lipoprotein; TG triglycerides; HDL high-density lipoprotein; $h s C R P$ high sensitivity $C$-reactive protein; VIF variance infiltration factor

were all $<4.0$ indicated that there exist no significant interactions between the variables (Table 1). In the whole population, $\mathrm{MS}(\mathrm{HR}=2.128,95 \% \mathrm{CI}: 1.524-2.970, P<$ $0.001)$, presence of high-risk plaques $(\mathrm{HR}=11.059$, 95\%CI: 7.749-57.232, $P<0.001)$ and hsCRP $(\mathrm{HR}=1.629$, 95\%CI: $1.128-2.352, P=0.009)$ were related to an increased risk of MACEs in patients with risk factors for coronary heart disease. Of the metabolic syndrome components, abdominal obesity $(\mathrm{HR}=1.264,95 \% \mathrm{CI}: 0.823-$ $0.908, P=0.033)$, hyperglycemia $(\mathrm{HR}=1.567,95 \% \mathrm{CI}$ : 1.096-2.639, $P=0.015)$, high blood pressure $(\mathrm{HR}=$ 1.700, 95\%CI:0.297-0.728, $P=0.018)$ and hyperlipidemia $(\mathrm{HR}=1.634,95 \% \mathrm{CI}: 0.431-0.933, P=0.021)$ were related to an increased risk of MACEs at 36 months. (Table 3).

Adjusted for statistically significant variables in Table 2 such as age, sex, BMI, waist circumference, HDL, TG, FBG, hsCRP, positive remodeling, spotty calcification, napkin ring sign, $\mathrm{MS}(\mathrm{HR}=2.265,95 \% \mathrm{CI}$ : $1.629-3.150, P<0.001)$ and hsCRP (HR $=1.267,95 \% \mathrm{CI}$ : $1.191-1.348, P=0.004)$ remained independent risk factors for MACEs in patients with high-risk coronary plaques at 36 months. Of the metabolic syndrome components, abdominal obesity $(\mathrm{HR}=$ 1.526, 95\%CI:1.118-2.082, $P=0.008)$, hyperglycemia ( $\mathrm{HR}=$ 1.640, 95\%CI:0.460-0.890, $P=0.003$ ) and high blood pressure $(\mathrm{HR}=1.405$, 95\%CI:0.264-0.620, $P<0.001)$ were related to an increased risk of MACEs. (Table 4). We also tested the proportional hazard assumption by using Schoenfeld residual test and found no breach of that hypothesis $(P=0.116)$; Variance inflation factors were all $<4.0$ so that there exist no significant interactions between the variables. (Table 2) Fig. 2 shows the number of patients with and without high-risk plaques according to the number of components of the metabolic syndrome they exhibited. As the number of MS components increased, the ratio of patients with high-risk plaques increased relative to the number without high-risk plaques.

Kaplan-Meier survival curve analysis showed statistically significant differences in MACEs between the MS and non-MS groups in the whole population over 36 months (log rank $P<0.0001$, Fig. 3). In the population with high-risk plaques there was also a significant difference in MACEs between the MS and non-MS groups over 36 months ( $\log$ rank $P<0.0001$, Fig. 4).

CCTA images from an example patient are presented in Fig. 5. The case was a 58-year-old male with metabolic syndrome, and the high-risk plaques (Fig. $5 \mathrm{a}-\mathrm{b}$ ) developed into severe coronary stenosis (Fig. $5 \mathrm{c}-\mathrm{d}$ ) in 32 months.

\section{Discussion}

This study aimed to analyze the prevalence of high-risk coronary plaques in patients with and without MS by CCTA and to investigate the relationship between MS, 
Table 2 Baseline clinical and biochemical characteristics in subjects with high risk plaques in the MS group and non-MS group

\begin{tabular}{|c|c|c|c|c|}
\hline & MS with high-risk plaques $(n=326)$ & Non-MS with high-risk plaques $(n=378)$ & $P$ & VIF \\
\hline Age (years), mean $\pm S D$ & $62 \pm 10.4$ & $61 \pm 11.5$ & 0.288 & 1.137 \\
\hline Male, n (\%) & $169(52)$ & $188(50)$ & 0.577 & 1.173 \\
\hline $\mathrm{BMI}\left(\mathrm{kg} / \mathrm{cm}^{2}\right)$, mean $\pm \mathrm{SD}$ & $26.8 \pm 3.67$ & $24.7 \pm 3.35$ & 0.032 & 1.440 \\
\hline Waist circumference $(\mathrm{cm})$, mean \pm SD & $91.8 \pm 6.80$ & $88.7 \pm 6.34$ & 0.026 & 1.348 \\
\hline Hypertension, n (\%) & $220(67.5)$ & $219(58.0)$ & 0.010 & 1.148 \\
\hline Diabetes, n (\%) & $217(66.6)$ & $207(54.8)$ & 0.002 & 1.138 \\
\hline Hyperlipidemia, n (\%) & $204(62.6)$ & 199 (52.6) & 0.010 & 1.608 \\
\hline $\mathrm{FBG}(\mathrm{mmol} / \mathrm{L})$, mean $\pm \mathrm{SD}$ & $6.7 \pm 2.66$ & $6.3 \pm 2.27$ & 0.006 & 1.125 \\
\hline $\mathrm{LDL}(\mathrm{mmol} / \mathrm{l})$, mean $\pm \mathrm{SD}$ & $3.5 \pm 0.78$ & $3.4 \pm 0.85$ & 0.248 & 1.629 \\
\hline $\mathrm{TG}(\mathrm{mmol} / \mathrm{l})$, mean $\pm \mathrm{SD}$ & $1.8 \pm 1.16$ & $1.6 \pm 0.33$ & 0.020 & 1.043 \\
\hline $\mathrm{HDL}(\mathrm{mmol} / \mathrm{l})$, mean $\pm \mathrm{SD}$ & $1.2 \pm 0.99$ & $1.4 \pm 0.74$ & 0.043 & 1.080 \\
\hline hsCRP (mg/l), mean $\pm \mathrm{SD}$ & $8.0 \pm 2.72$ & $7.1 \pm 3.15$ & $<0.001$ & 1.950 \\
\hline \multicolumn{5}{|l|}{ Pharmacological treatment, n (\%) } \\
\hline Hypertension, n (\%) & $141(43.3)$ & $154(40.7)$ & 0.501 & 1.144 \\
\hline Diabetes, n (\%) & $123(37.7)$ & $118(31.3)$ & 0.069 & 1.367 \\
\hline Hyperlipidemia, n (\%) & $81(24.8)$ & $76(20.1)$ & 0.132 & 1.021 \\
\hline Aspirin, n (\%) & $173(53.1)$ & $177(46.8)$ & 0.112 & 1.239 \\
\hline Statins, n(\%) & $94(28.9)$ & $93(24.7)$ & 0.231 & 1.069 \\
\hline Positive remodeling, n (\%) & $216(66.3)$ & $205(54.2)$ & 0.001 & 1.440 \\
\hline Low attenuation, n (\%) & $142(43.6)$ & $155(41.0)$ & 0.540 & 1.148 \\
\hline Spotty calcification, n (\%) & $213(65.3)$ & $207(54.8)$ & 0.004 & 1.264 \\
\hline Napkin ring sign, n (\%) & $165(50.6)$ & $154(40.7)$ & 0.010 & 2.203 \\
\hline MACEs, n (\%) & $96(29)$ & $57(15)$ & $<0.001$ & \\
\hline
\end{tabular}

MS metabolic syndrome; $B M I$ body mass index; $F B G$ fasting blood glucose; $L D L$ low-density lipoprotein; $T G$ triglycerides; $H D L$ high-density lipoprotein; $h s C R P$ high sensitivity C-reactive protein; VIF variance infiltration factor

high-risk coronary plaques, and MACEs. The results showed that there were more high-risk plaques and higher rates of MACEs in the MS group compared to the non-MS group. When the population with high-risk plaques was considered alone, MACEs remained higher in the MS group; hsCRP and the ratio of positive remodeling, spot-like calcification and napkin ring signs of the imaging characteristics of high-risk plaques in the MS group were significantly higher than that in non-MS group. COX regression analysis in the whole population showed metabolic syndrome, presence of a high-risk coronary plaque and abnormal hsCRP were risk factors for MACEs at 36 months. Of the metabolic syndrome components, abdominal obesity, hyperglycemia, high blood pressure and hyperlipidemia were all risk factors. Metabolic syndrome remained an independent risk

Table 3 Multivariate COX regression analysis of risk factors for MACEs events in all patients (No. =1136) at 36 months

\begin{tabular}{|c|c|c|c|}
\hline & $H R$ & $95 \% \mathrm{Cl}$ & P \\
\hline Metabolic syndrome, Yes vs. No & 2.128 & $1.524-2.970$ & $<0.001$ \\
\hline \multicolumn{4}{|l|}{ Metabolic components } \\
\hline Abdominal obesity, Yes vs. No & 1.264 & $0.823-0.908$ & 0.033 \\
\hline Hyperglycemia, Yes vs. No & 1.567 & $1.096-2.639$ & 0.015 \\
\hline High blood pressure, Yes vs. No & 1.700 & $0.297-0.728$ & 0.018 \\
\hline Hyperlipidemia, Yes vs. No & 1.634 & $0.431-0.933$ & 0.021 \\
\hline Presence of high-risk coronary plaque, Yes vs. No & 11.059 & $7.749-57.232$ & $<0.001$ \\
\hline hsCRP, $>10 \mathrm{mg} / \mathrm{l}$ VS $\leq 10 \mathrm{mg} / \mathrm{L}$ & 1.629 & $1.128-2.352$ & 0.009 \\
\hline
\end{tabular}

Adjusted for age, sex, BMI, waist circumference, LDL, TG, FBG 
Table 4 Multivariate COX regression analysis of risk factors for MACEs in patients with high risk plaques (No. $=704)$ at 36 months

\begin{tabular}{llll}
\hline & HR & $95 \% \mathrm{Cl}$ & $\mathrm{P}$ \\
\hline Metabolic syndrome, Yes vs. No & 2.265 & $1.629-3.150$ & $<0.001$ \\
Metabolic components & & & \\
Abdominal obesity, Yes vs. No & 1.526 & $1.118-2.082$ & 0.008 \\
Hyperglycemia, Yes vs. No & 1.640 & $0.460-0.890$ & 0.003 \\
High blood pressure, Yes vs. No & 1.405 & $0.264-0.620$ & $<0.001$ \\
Hyperlipidemia, Yes vs. No & 1.277 & $0.898-1.817$ & 0.174 \\
hsCRP, >10 mg/l VS $\leq 10 \mathrm{mg} / \mathrm{L}$ & 1.267 & $1.191-1.348$ & 0.004 \\
\hline
\end{tabular}

Adjusted for age, sex, BMI, waist circumference, HDL, TG, FBG, hsCRP, positive remodeling, spotty calcification, napkin ring sign

factor for MACEs in patients with high-risk coronary plaques at 36 months and the other related risk factors were hsCRP, abdominal obesity, hyperglycemia, and high blood pressure. Kaplan-Meier survival curve analysis showed statistically significant differences in MACEs between the MS and non-MS groups in the whole population over 36 months and in the population with high-risk plaques.

Previous study has shown that metabolic syndrome has a relationship with atherosclerosis [4, 24]. In particular, the previous studies found the abdominal obesity and high blood pressure [4] or waist circumference and insulin resistance [25] components of metabolic syndrome were significantly associated with coronary plaques. In this study we found that the rate of high-risk plaques was higher in patients with metabolic syndrome. However, another study that used duplex ultrasound to investigate carotid plaque morphology in asymptomatic patients with and without metabolic syndrome found that metabolic syndrome did not affect the stenosis grade or the rate of unstable carotid plaques [25]. This study suggests that metabolic syndrome is more likely to lead to unstable plaques.
Insulin resistance is the central link of metabolic syndrome, and hsCRP concentration has been shown to be closely related to insulin resistance. It has been reported that serum hsCRP level of patients with cardiovascular disease combined with insulin resistance is higher than that of patients without insulin resistance, and it is positively correlated with saturated fatty acids and negatively correlated with unsaturated fatty acids [26]. Patients with high hsCRP level have more thin fibrous caps detected by optical correlation tomography (OCT), suggesting that hsCRP can reflect the instability of coronary plaque [27]. In this study, when the subpopulation of patients was investigated, the MS group had higher levels of hsCRP suggesting higher levels of inflammation and oxidative stress in patients with metabolic syndrome and providing a suitable pathophysiological environment for the development of high-risk plaques.

Based on characteristics such as low CT attenuation, napkin ring sign, positive remodeling and spotty calcification by CCTA, the high-risk plaques are vulnerable to rupture. The lesions with positive remodeling were larger in size and had more necrotic centers [28]. Spotty calcification tends to worsen the condition of patients with stable angina pectoris [29]. Napkin ring sign is the result of comparison between large necrotic centers (low central attenuation) and fibrous plaque tissues (slightly higher annular attenuation). In CT examination, napkin ring sign is used to identify severe coronary artery plaques and plaques covered with thin fibrous caps with high specificity, which can independently predict MACEs [30]. In patients with high-risk plaques in this study, the proportion of positive remodeling, spotty calcification and napkin ring sign in the MS group was significantly higher than that in the non-MS group, while there was no significant difference between the two groups with low CT attenuation, suggesting that metabolic syndrome has more influence on the size of high-

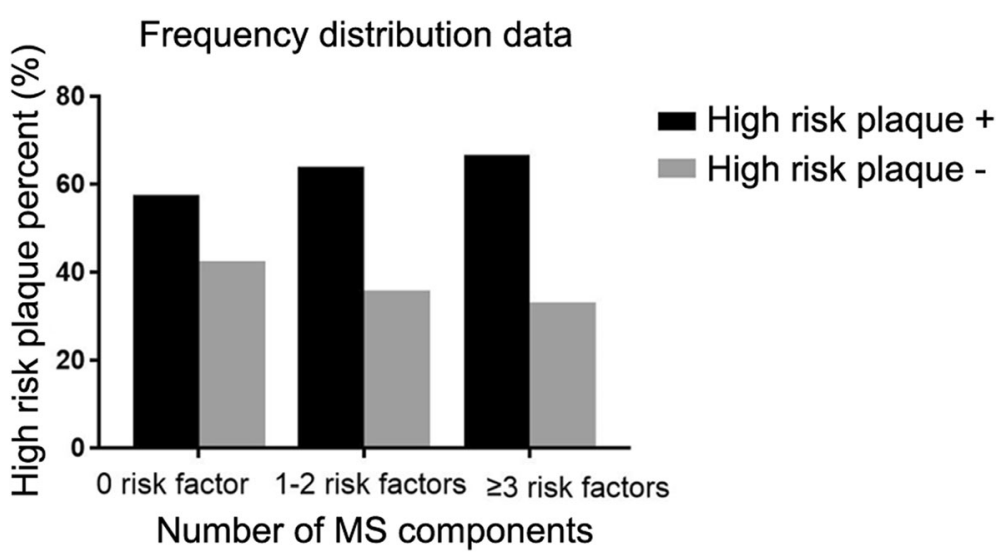

Fig. 2 Graph showing the percentage of patients with and without high-risk plaques according to their number of metabolic syndrome components. The prevalence of high-risk coronary plaques increased as the number of metabolic syndrome components increased 
MACEs between MS group and non-MS group in

all patients by following up for $\mathbf{3 6}$ months

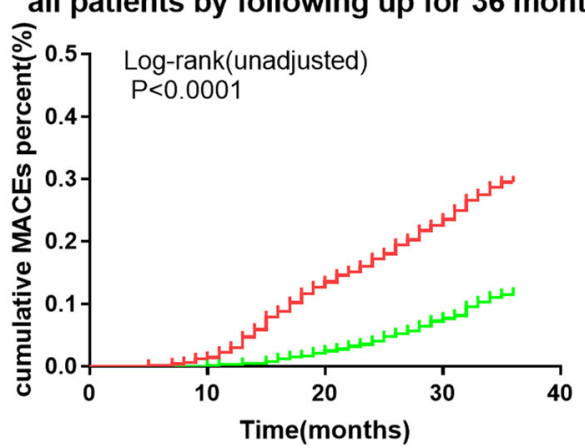

- MS group

+ non-MS group

Patient at risk

$\begin{array}{llllll}\text { MS group } & 488 & 465 & 410 & 354 & 344 \\ \text { non-MS group } & 648 & 646 & 625 & 581 & 573\end{array}$

Fig. 3 Kaplan-Meier survival curve showing MACEs in all patients followed up for 36 months

risk plaques, the number of necrotic centers and the degree of calcification rather than the density of lipid in plaques. We are unsure why there is a difference between our results and the other study, but the patients with metabolic syndrome in the previous study used more classes of antihypertensive drugs and were treated with statins for a longer period than the patients without metabolic syndrome, so this may have caused some bias in the results. There are differences between the two studies in terms of the patient populations, because our study was in a Chinese population and the other study was undertaken in Brazil [25]. Also, there are some differences in the plaque analysis between these two studies. However, further investigation is needed to fully investigate the different conclusions of the studies. During follow-up the proportion of MACEs in patients with metabolic syndrome was significantly increased, and multiple regression analysis still indicated that metabolic syndrome was a risk factor for MACEs after adjustment for the relevant risk factors. This indicates that the calcification score of patients with metabolic syndrome progresses rapidly and is an independent risk factor for the progression of high-risk vulnerable coronary plaques [31]. Metabolic syndrome also increases the risk of coronary atherosclerosis in postmenopausal women through arterial stiffness [32]. In patients with metabolic syndrome, high-risk plaques are larger in size, have more thin fibrous caps and necrotic centers, and their higher levels of inflammation and oxidative stress can increase the instability of plaques. High-risk plaques are prone to rapid progress, rupture and induce

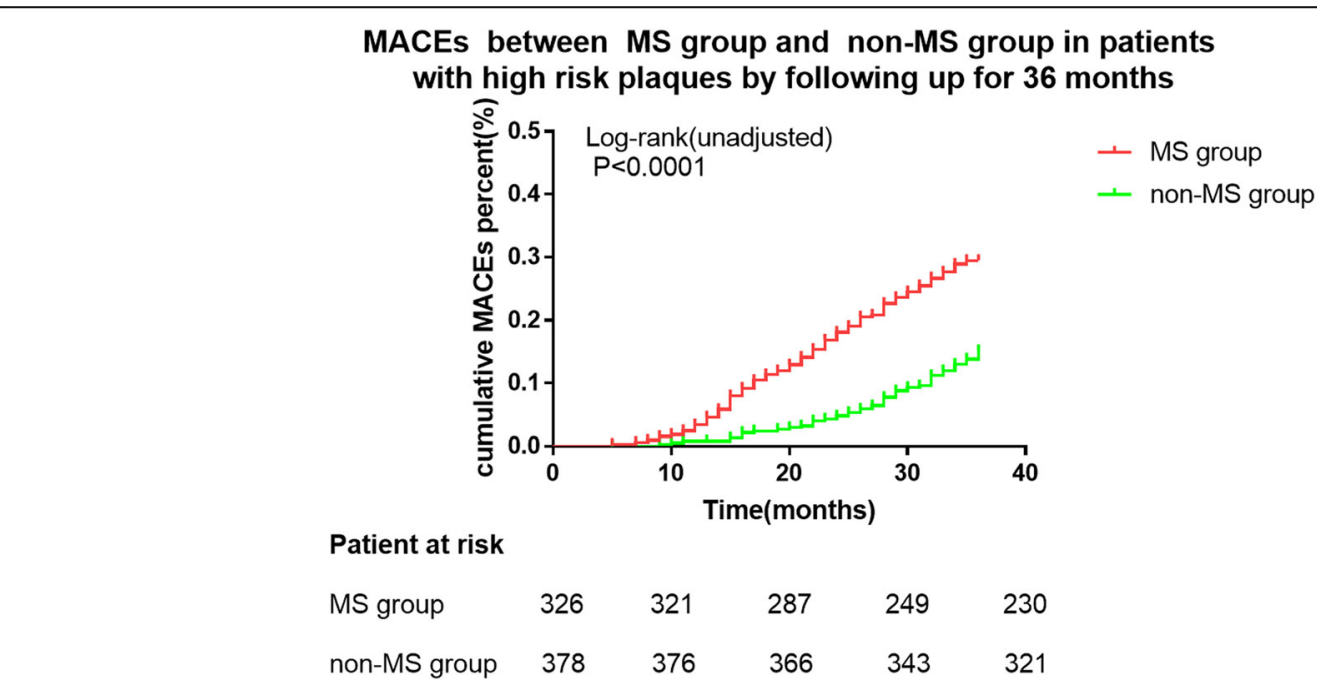

Fig. 4 Kaplan-Meier survival curve showing MACEs of patients with high risk plaques followed up for 36 months 
A
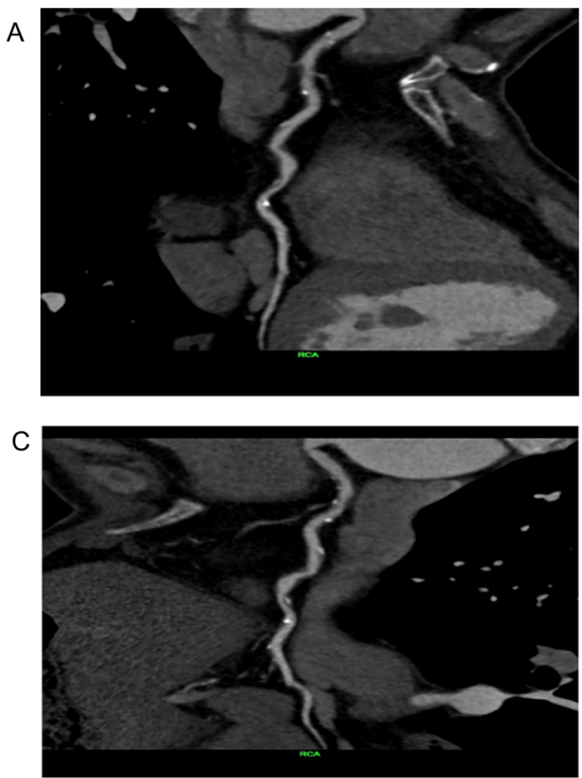

B

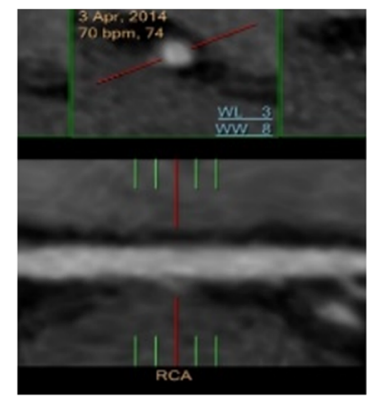

D

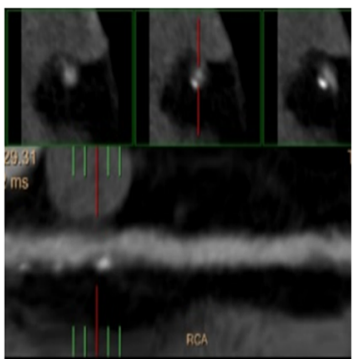

Fig. 5 A 58-year-old male patient with metabolic syndrome. Mild stenosis was found in the right coronary artery by CCTA in April 2014, and the mixed plaque was recognized as high-risk plaque (positive remodeling, spotty calcification) (a - b). After 32 months, the patient underwent CCTA again due to unstable angina pectoris. The stenosis in right coronary artery developed into $80 \%$ in the proximal and $50 \%$ in the middle (c - d)

coronary events. However, patients with metabolic syndrome themselves may have a higher plaque load and the number of high-risk plaques.

This study has some limitations. The study was quite small and from one hospital. A larger study would add more evidence for these results. As CCTA is not widely used in clinical screening for coronary heart disease in asymptomatic people, the relationship between high-risk plaques and metabolic syndrome in asymptomatic people was not included in this study, which may cause bias to the results. Besides, there is no evaluation of the cause of high-risk plaques. Whether metabolic syndrome is more likely to increase the vulnerability of a single plaque cannot be inferred, this requires further studies on dynamic change of single high-risk plaque.

\section{Conclusions}

Comparison of patients with chest pain with and without metabolic syndrome showed that high-risk plaques were more common in patients with metabolic syndrome. Metabolic syndrome, in particular abdominal obesity, high blood pressure, and hyperlipidemia, and the presence of high-risk coronary plaques were all risk factors for MACEs. In patients with high-risk plaques MACEs related risk factors were hsCRP and metabolic syndrome, in particular abdominal obesity, hyperglycemia, and high blood pressure. Studies on the impact of metabolic syndrome on single high-risk plaque and the effect of every metabolic syndrome component on the prognosis of high-risk plaque are still needed. Subgroup analysis based on gender, age also should be discussed in future research.

\section{Abbreviations}

AS: Atherosclerosis; BMI: Body mass index; CCTA: Coronary computed tomography angiography; CPR: Curved planar reconstruction; FBG: Fasting blood glucose; HDL: High-density lipoprotein; hsCRP: High sensitivity Creactive protein; HU: Hounsfield unit; LDL: Low-density lipoprotein; MACEs: Major adverse cardiovascular events; MIP: Maximum intensity projection; MPR: Multi-layer reconstruction; MS: Metabolic syndrome; SD: Standard deviation; STEMI: ST-elevation myocardial infarction; TG: Triglycerides; UA/NSTE-MI: Unstable angina/non-STEMI; VIF: Variance infiltration factor; VR: Volume rendering

\section{Acknowledgements \\ Not applicable.}

\section{Authors' contributions}

$Z R$ and $Y X$ conceived and supervised the study; YX and LXW designed experiments; ZH and LS performed CCTA analysis; LW, HS, ZL and ZJ performed information acquisition and examination analysis; $Y X, L W, H S, Z L$, ZJ and LXW performed follow-up, YX wrote the manuscript; $Y X$ and ZR made manuscript revisions. All authors reviewed the results and approved the final version of the manuscript.

\section{Funding}

This study was supported by the 2014 Tianjin Municipal Health Planning Commission key research project (No.14KG126). The funders had no role in study design, data collection and analysis, decision to publish, or preparation of the manuscript.

\section{Availability of data and materials}

The datasets used and/or analysed during the current study are available from the corresponding author on reasonable request.

\section{Ethics approval and consent to participate}

This study was approved by Ethic Committees of the Tianjin Chest Hospital. The ethical code of the study was 2019LW-006. All patients underwent multidetector $C T$ and had provided written informed consent after they had agreed to participate in our study. 


\section{Consent for publication}

Not applicable.

\section{Competing interests}

The authors declare that they have no competing interests.

\section{Author details}

'Department of Cardiology, Tianjin Chest Hospital, Tianjin 300222, People's Republic of China. ${ }^{2}$ Department of Radiology, Tianjin Chest Hospital, Tianjin 300222, People's Republic of China.

\section{Received: 9 August 2019 Accepted: 24 January 2020}

Published online: 11 February 2020

\section{References}

1. O'Neill S, O'Driscoll L. Metabolic syndrome: a closer look at the growing epidemic and its associated pathologies. Obes Rev. 2015;16:1-12.

2. Lu J, Wang L, Li M, Xu Y, Jiang Y, Wang W, et al. Metabolic syndrome among adults in China: the 2010 China noncommunicable disease surveillance. J Clin Endocrinol Metab. 2017;102:507-15.

3. Hess PL, Al-Khalidi HR, Friedman DJ, Mulder H, Kucharska-Newton A, Rosamond WR, et al. The metabolic syndrome and risk of sudden cardiac death: the atherosclerosis risk in communities study. J Am Heart Assoc. 2017;6(8). https://doi.org/10.1161/JAHA.117.006103.

4. Ryu J, Yong HS, Huh S, Kang EY, Woo OH. Relation of coronary atherosclerosis and metabolic syndrome in asymptomatic subjects: evaluation with coronary CT angiography. Int J Cardiovasc Imag. 2013;29(Suppl 2):101-7.

5. Park YM, Han SH, Seo JG, Lee S, Oh PC, Koh KK, et al. The role of insulin resistance and metabolic risk factors on culprit coronary plaque. Int J Cardiol. 2015;190:56-62.

6. Thomsen C, Abdulla J. Characteristics of high-risk coronary plaques identified by computed tomographic angiography and associated prognosis: a systematic review and meta-analysis. Eur Heart J Cardiovasc Imaging. 2016;17:120-9.

7. Maurovich-Horvat P, Ferencik M, Voros S, Merkely B, Hoffmann U. Comprehensive plaque assessment by coronary $\mathrm{CT}$ angiography. Nat Rev Cardiol. 2014;11:390-402.

8. Tomizawa N, Yamamoto K, Hayakawa Y, Inoh S, Nojo T, Nakamura S. A clinical model to identify patients with high-risk plaque by coronary computed tomography angiography. Int J Cardiol. 2017;228:260-4.

9. Szilveszter B, Celeng C, Maurovich-Horvat P. Plaque assessment by coronary CT. Int J Cardiovasc Imag. 2016:32:161-72.

10. Ahmadi A, Leipsic J, Feuchtner G, Gransar H, Kalra D, Heo R, et al. Is metabolic syndrome predictive of prevalence, extent, and risk of coronary artery disease beyond its components? Results from the multinational coronary CT angiography evaluation for clinical outcome: an international multicenter registry (CONFIRM). PLoS One. 2015;10:e0118998.

11. Wilson PW, Kannel WB, Silbershatz H, D'Agostino RB. Clustering of metabolic factors and coronary heart disease. Arch Intern Med. 1999:159:1104-9.

12. Synetos A, Papanikolaou A, Toutouzas K, Georgiopoulos G, Karanasos A, Drakopoulou $\mathrm{M}$, et al. Metabolic syndrome predicts plaque rupture in patients with acute myocardial infarction. An optical coherence study. Int J Cardiol. 2016:209:139-41.

13. Chirinos DA, Medina-Lezama J, Arguelles W, Goldberg R, Schneiderman N, Khan Z, et al. Metabolic syndrome as an underlying disease entity and its relationship to subclinical atherosclerosis in Andean Hispanics. Metab Syndr Relat Disord. 2014;12:49-55.

14. van Rooy MJ, Pretorius E. Metabolic syndrome, platelet activation and the development of transient ischemic attack or thromboembolic stroke. Thromb Res. 2015:135:434-42.

15. Szentpetery A, Healy GM, Brady D, Haroon M, Gallagher P, Redmond CE, et al. Higher coronary plaque burden in psoriatic arthritis is independent of metabolic syndrome and associated with underlying disease severity. Arthritis Rheumatol. 2018;70:396-407.

16. Yonetsu T, Kato K, Uemura S, Kim BK, Jang Y, Kang SJ, et al. Features of coronary plaque in patients with metabolic syndrome and diabetes mellitus assessed by 3vessel optical coherence tomography. Circ Cardiovasc Imag. 2013;6:665-73.

17. Expert Panel on Detection E, Treatment of High Blood Cholesterol in A. Executive Summary of The Third Report of The National Cholesterol Education Program (NCEP) Expert Panel on Detection, Evaluation, And Treatment of High Blood Cholesterol In Adults (Adult Treatment Panel III). JAMA. 2001;285:2486-97.
18. Grundy SM, Cleeman JI, Daniels SR, Donato KA, Eckel RH, Franklin BA, et al. Diagnosis and management of the metabolic syndrome: an American Heart Association/National Heart, Lung, and Blood Institute scientific statement: executive summary. Crit Pathw Cardiol. 2005:4:198-203.

19. American College of Cardiology Foundation/American Heart Association/ American College of Physicians Task Force on Clinical C, Training, Society of Atherosclerosis I, Prevention, Society for Cardiovascular A, Interventions, et al. ACCF/AHA 2007 clinical competence statement on vascular imaging with computed tomography and magnetic resonance: a report of the American College of Cardiology Foundation/American Heart Association/ American College of Physicians Task Force on Clinical Competence and Training: developed in collaboration with the Society of Atherosclerosis Imaging and Prevention, the Society for Cardiovascular Angiography and Interventions, the Society of Cardiovascular Computed Tomography, the Society for Cardiovascular Magnetic Resonance, and the Society for Vascular Medicine and Biology. Circulation. 2007;116:1318-35.

20. Conte E, Annoni A, Pontone G, Mushtaq S, Guglielmo M, Baggiano A, et al. Evaluation of coronary plaque characteristics with coronary computed tomography angiography in patients with non-obstructive coronary artery disease: a long-term follow-up study. Eur Heart J Cardiovasc Imaging. 2017;18:1170-8.

21. $M J K, S L J, D R B, O P M, W J W, R$ DJ, et al. Prognostic value of multidetector coronary computed tomographic angiography for prediction of all-cause mortality, vol. 50; 2007. p. 1161-70.

22. $H$ M, A S, M A-M, B D, B M, C F, et al. Optimized prognostic score for coronary computed tomographic angiography: results from the CONFIRM registry (COronary CT Angiography EvaluatioN For Clinical Outcomes: An InteRnational Multicenter Registry). J Am Coll Cardiol. 2013;62:468-76.

23. Eckel RH, Jakicic JM, Ard JD, de Jesus JM, Houston Miller N, Hubbard VS, et al. 2013 AHA/ACC guideline on lifestyle management to reduce cardiovascular risk: a report of the American College of Cardiology/ American Heart Association task force on practice guidelines. J Am Coll Cardiol. 2014;63:2960-84.

24. Konishi M, Sugiyama S, Sugamura K, Nozaki T, Ohba K, Matsubara J, et al. Total coronary artery plaque burden measured by cardiac computed tomography is associated with metabolic syndrome. J Atheroscler Thromb. 2011;18:939-45.

25. Cury MV, Presti C, Bonadiman SS, Casella IB, Benabou JE, da Silva ES, et al. Carotid plaque morphology in asymptomatic patients with and without metabolic syndrome. Ann Vasc Surg. 2017;39:173-81.

26. Bersch-Ferreira AC, Sampaio GR, Gehringer MO, Torres E, Ross-Fernandes MB, da Silva JT, et al. Association between plasma fatty acids and inflammatory markers in patients with and without insulin resistance and in secondary prevention of cardiovascular disease, a cross-sectional study. Nutr J. 2018;17:26.

27. Koyama K, Yoneyama K, Mitarai T, Ishibashi Y, Takahashi E, Kongoji K, et al. Association between inflammatory biomarkers and thin-cap fibroatheroma detected by optical coherence tomography in patients with coronary heart disease. Arch Med Sci. 2015;11:505-12.

28. Kroner ES, van Velzen JE, Boogers MJ, Siebelink HM, Schalij MJ, Kroft LJ, et al. Positive remodeling on coronary computed tomography as a marker for plaque vulnerability on virtual histology intravascular ultrasound. Am J Cardiol. 2011;107:1725-9.

29. Ferencik M, Schlett CL, Ghoshhajra BB, Kriegel MF, Joshi SB, MaurovichHorvat $\mathrm{P}$, et al. A computed tomography-based coronary lesion score to predict acute coronary syndrome among patients with acute chest pain and significant coronary stenosis on coronary computed tomographic angiogram. Am J Cardiol. 2012;110:183-9.

30. Maurovich-Horvat P, Schlett CL, Alkadhi H, Nakano M, Otsuka F, Stolzmann $P$, et al. The napkin-ring sign indicates advanced atherosclerotic lesions in coronary CT angiography. JACC CardiovasC Imaging. 2012;5:1243-52.

31. Kim LK, Yoon JW, Lee DH, Kim KM, Choi SH, Park KS, et al. Impact of metabolic syndrome on the progression of coronary calcium and of coronary artery disease assessed by repeated cardiac computed tomography scans. Cardiovasc Diabetol. 2016;15:92.

32. Lambrinoudaki I, Kazani A, Armeni E, Rizos D, Augoulea A, Kaparos G, et al. The metabolic syndrome is associated with carotid atherosclerosis and arterial stiffness in asymptomatic, nondiabetic postmenopausal women. Gynecol Endocrinol. 2018;34:78-82.

\section{Publisher's Note}

Springer Nature remains neutral with regard to jurisdictional claims in published maps and institutional affiliations. 